\title{
EVALUATING THE EFFECTS OF MAGNETIC SUSCEPTIBILITY IN UXO DISCRIMINATION PROBLEMS
}

\author{
Leonard R. Pasion, Stephen D. Billings, and Douglas W. Oldenburg \\ $U B C$ - Geophysical Inversion Facility \\ Department of Earth and Ocean Sciences, University of British Columbia \\ Vancouver, B.C., V6T 1Z4, CANADA \\ email: pasion@geop.ubc.ca
}

\begin{abstract}
Past unexploded ordnance (UXO) clearance projects have demonstrated that the ability to detect UXO can be seriously hindered by the presence of magnetic rocks and soils. In particular, magnetic material affects the decay curve characteristics in electromagnetic surveys and this can adversely affect inversion calculations that try to estimate parameters of the UXO or answer the question of whether the item is UXO or scrap. Our research is in two parts. Firstly, we carry out numerical 1D forward modelling to evaluate the distortions of signals due to laterally uniform magnetic material. In the second part of the talk we examine how the distortions in the signal affect our inversion algorithms used for UXO discrimination.
\end{abstract}

\section{Introduction}

The task of discriminating UXO from non-UXO items is more difficult when sensor data is contaminated with geological noise originating from magnetic soils. The magnetic properties of soils are mainly due to the presence of iron. Hydrated iron oxides such as muscovite, dolomite, lepidocrocite, and geothite are weakly paramagnetic, and play a minor role in determining the magnetic character of the soil. The magnetic character of the soil is dominated by the presence of ferrimagnetic minerals such as maghaemite $\left(\alpha \mathrm{Fe}_{2} \mathrm{O}_{4}\right)$ and magnetite $\left(\mathrm{Fe}_{3} \mathrm{O}_{4}\right)$. Maghaemite is considered the most important of the minerals within archaeological remote sensing circles (for example [Scollar et al., 1990]). Magnetite is the most magnetic of the iron oxides, and is the most important mineral when considering the effects of magnetic soils on EM measurements.

Electromagnetic sensors are sensitive to the presence of magnetite due to the phenomenon of magnetic viscosity. Electromagnetic sensors illuminate the subsurface with a time or frequency varying primary field. Suppose that we apply a magnetic field $H$ to an area containing magnetic soil. The magnetization vector of the soils will try to adjust to align itself with the exciting field. At the instant the magnetic field is applied there is an immediate change in magnetization and, possibly, an additional time dependent change in magnetization. This time dependent phenomenon is referred to as magnetic viscosity or magnetic after-effect. A time constant $\tau$ is used to characterize the time for the magnetization vector to rotate from its minimum energy orientation prior to application of the field, to its new orientation. For a sample of magnetic grains which has a large range of relaxation times that are 
distributed uniformly over their spectrum, the magnetic moment of the soil sample will decay logarithmically [Chikazumi, 1997]. Consequently, the time derivative of the decaying magnetic field produced by the magnetization decays as $t^{-1}$. This $t^{-1}$ decay have been observed in archaeological prospecting [Colani and Aitken, 1996], time domain electromagnetic (TEM) surveys carried out over laeteritic soils for mineral exploration [Buselli, 1982], and also in TEM surveys carried out on Kaho'olawe Island, Hawaii [Ware, 2001].

This talk focuses on the effects of magnetic soils and magnetic viscosity on time domain and frequency domain electromagnetic sensor data. By forward modelling of a 1-D layered earth model we hope to obtain some sense of how sensitive surveys are to magnetic soils, and the strength of the magnetic soil response relative to buried metallic objects. We then see how perturbations in signal due to magnetic soil affect the ability to recover parameters of the dipole model presented in Pasion and Oldenburg [2001a].

\section{Electromagnetic Response of Magnetic Soils}

\section{Frequency Dependent Susceptibility Models}

Lee [1983] showed for that a sample containing a collection of particles with a uniform distribution of decay times has a magnetic susceptibility of

$$
\chi=\chi_{o}\left[1-\frac{1}{\ln \left(\tau_{2} / \tau_{1}\right)} \ln \left(\frac{i \omega \tau_{2}+1}{i \omega \tau_{1}+1}\right)\right]
$$

This model of susceptibility is expressed as a function of two time constants ( $\tau_{1}$ and $\tau_{2}$ ) that determine the limits of the uniform distribution of time constants, and the static $(\omega=0)$ susceptibility $\chi_{0}$. The

quadrature and inphase components described by this model are plotted in Figure 1(a). Between the frequencies $\omega=1 / \tau_{2}$ and $\omega=1 / \tau_{1}$ the quadrature component of susceptibility is nearly constant, but there is a peak at $\omega_{m}=\left(\tau_{1} \tau_{2}\right)^{-1}$. The inphase component decreases linearly with the logarithm of frequency.

The Cole-Cole model has also been used to represent the magnetic susceptibility (for example [Olhoeft and Strangway, 1974] and [Dabas et al., 1992]). Figure 1(b) contains plots of the Cole-Cole distribution for a range of distributions. The Cole-Cole model for magnetic susceptibility is:

$$
\chi=\chi_{\infty}+\frac{\chi_{o}-\chi_{\infty}}{1+(i \omega \tau)^{1-\alpha}}
$$

where $\chi_{\infty}$ is the real susceptibility as frequency approaches infinity $(\omega \rightarrow \infty)$ and $\chi_{0}$ is the real susceptibility as frequency approaches zero $(\omega \rightarrow 0)$. We assume that $\chi_{\infty}=0$ and that the model for magnetic susceptibility can be written as

$$
\chi=\frac{\chi_{o}}{1+(i \omega \tau)^{1-\alpha}}
$$


The parameter $\alpha$ is related to the distribution of relaxation times. The limits of $\alpha$ are $\alpha=0$ for a single Debye relaxation mechanism [Olhoeft and Strangway, 1974] and $\alpha=1$ for an infinitely broad distribution of relaxation times. A plot of Cole-Cole distributions for a range of $\alpha$ values is shown in Figure 1. For the broad range of distribution of relaxation times that we are assuming in this study, we

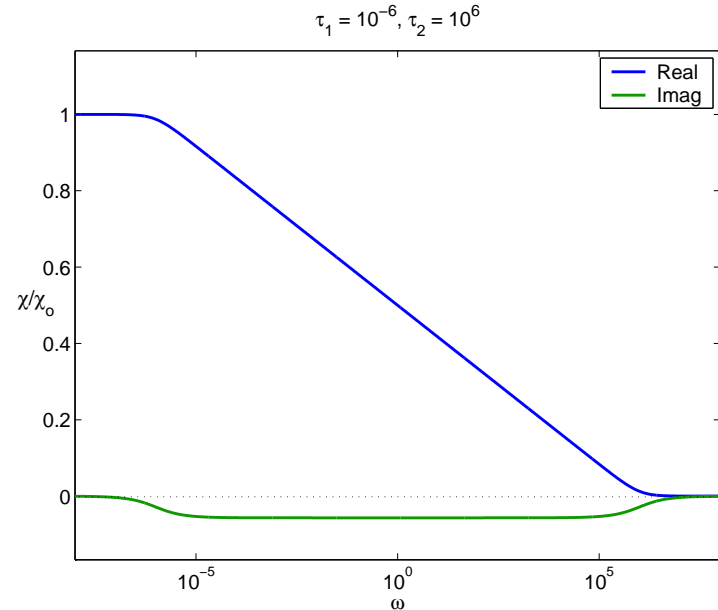

(a) Susceptibility Model from Lee (1983).

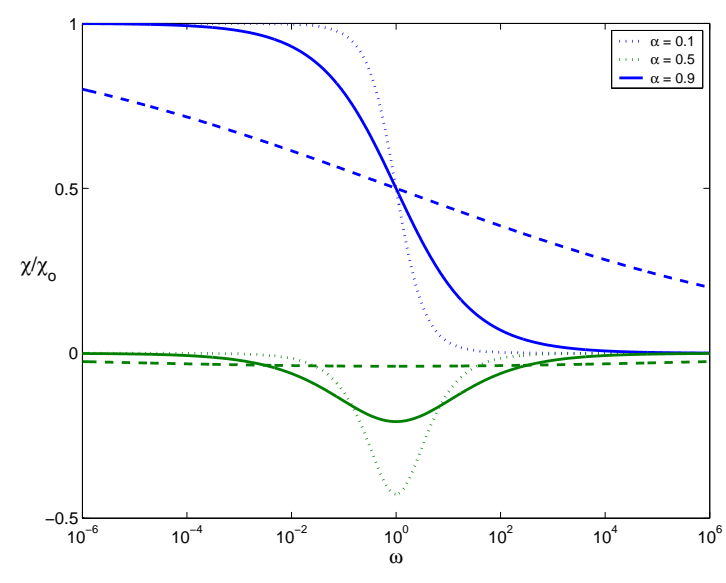

(b) Cole-Cole model of susceptibility as a function of $\alpha$. The time constant $\tau=1$.

Figure 1: Different frequency dependent susceptibility models.

would expect to model susceptibility with $\alpha$ values approaching 1 . The time constant $\tau$ controls the location of the peak of the imaginary part of the susceptibility, with the peak occurring at $\omega=1 / \tau$. For large $\alpha$ the Cole-Cole model yields a straight line for the real part and a constant negative value for the imaginary part. This is the same as Lee's representation and is a key characteristic for determining the behaviour of the electromagnetic response.

\section{Constructing a Magnetic Susceptibility Model from Kaho'olawe Soil Measurements}

The problems of magnetic soils at Kaho'olawe have been well documented [Cespedes, 2001]. A model for the frequency dependent susceptibility of Kaho'olawe soils is required to investigate magnetic noise problems through forward modelling. Kaho' olawe is a single volcanic dome made of thin bedded pahoehoe (smooth, very fast moving lava) and 'a' a (rugged, slow moving lava) basalt [Stearns, 1940]. The base rock is tholeiitic basalt, containing up to $20 \%$ magnetite [Butler, personal communication, 2001]. The base rock is covered by a number of different soil types with variable geophysical characteristics [Parsons-UXB, 1998].

Table 1 lists measurements of the magnetic susceptibility for soil samples at Seagull and Lua Kakika sites on Kaho'Olawe. These measurements provide us with the real part of the complex susceptibility at two frequencies. Given this limited information of the soil's magnetic characteristics, we need to make some assumptions in generating a susceptibility model. First, we assume that the two measuring 
(a) Seagull Site - Magnetic Susceptibility $\left(\times 10^{-5} \mathrm{SI}\right)$

\begin{tabular}{|l||c|c|c|}
\hline Sample & $\begin{array}{c}\text { Low Frequency } \\
(0.46 \mathrm{kHz})\end{array}$ & $\begin{array}{c}\text { High Frequency } \\
(4.6 \mathrm{kHz})\end{array}$ & $\begin{array}{c}\text { \% Frequency } \\
\text { Effect }\end{array}$ \\
\hline \hline 7462-2728A-6" & 3554 & 3311 & 7.0 \\
7462-2728A-24" & 3022 & 2771 & 8.2 \\
\hline 7462-2728B-6" & 1046 & 1001 & 4.3 \\
\hline 7468-2734AP-6" & 1726 & 1630 & 5.6 \\
7468-2734AP-18" & 1529 & 1448 & 5.3 \\
\hline 7468-2734BP-12" & 2807 & 2634 & 6.2 \\
7468-2734BP-24" & 1920 & 1807 & 5.9 \\
\hline 7468-2734AB-6" & 845 & 805 & 4.6 \\
\hline 7468-2734BB-8" & 1795 & 1707 & 4.9 \\
\hline
\end{tabular}

(b) Lua Kakika Site - Magnetic Susceptibility $\left(\times 10^{-5}\right.$ SI $)$

\begin{tabular}{|l||c|c|c|}
\hline Sample & $\begin{array}{c}\text { Low Frequency } \\
(0.46 \mathrm{kHz})\end{array}$ & $\begin{array}{c}\text { High Frequency } \\
(4.6 \mathrm{kHz})\end{array}$ & $\begin{array}{c}\text { \% Frequency } \\
\text { Effect }\end{array}$ \\
\hline \hline 7537-2754AP-6" & 2355 & 2249 & 4.5 \\
7537-2754AP-18" & 2227 & 2134 & 4.2 \\
\hline 7537-2754BP-12" & 1461 & 1383 & 5.4 \\
7537-2754BP-24" & 1497 & 1411 & 5.8 \\
\hline 7537-2754AB-6" & 2475 & 2431 & 1.8 \\
\hline 7537-2754B-12" & 1394 & 1334 & 4.3 \\
\hline
\end{tabular}

Table 1: Three separate samples were measured from each bag of soil. The mean of the measurements is listed.

frequencies are within the frequency range where the inphase component decreases linearly with the logarithm of frequency $\left(\tau_{2}^{-1} \ll \omega \ll \tau_{1}^{-1}\right)$. Second, we assume that all the frequencies of interest fall within this range of frequencies. With these two assumptions, we can model the real part of the susceptibility as a straight line. By manipulating equation 1 we can show that for $\tau_{2} \gg \tau_{1}$ the slope of the inphase component is related to the quadrature component by

$$
\text { Slope }=\left[\frac{\partial \operatorname{Re}(\chi)}{\partial \ln \omega}\right]=\frac{2}{\pi} \operatorname{Im}(\chi(\omega))=-\frac{\chi_{o}}{\ln \left(\tau_{2} / \tau_{1}\right)}
$$

This relationship has also been derived without the use of equation (1) [Mullins and Tite, 1974], and has been observed in complex susceptibility measurements by Dabas et al. [Dabas et al., 1992]. Therefore, by determining the slope of the inphase component from the susceptibility measurements, we can immediately estimate the quadrature component. The susceptibility model of Figure 2 was constructed using susceptibility measurements of sample 7462-2728A-6 (Table 1). In 1D earth modelling that follows, one of the layered earth models considered will use this magnetic susceptibility model to represent the susceptibility for the top layer of soil.

\section{Electromagnetic Response of a 1-D Layered Magnetic Earth}

Forward modelling in 1D is solved in the frequency domain in the standard propagation matrix formalism [Farquharson et al., 2001]. Let us consider a circular transmitter loop of radius $a$, carrying a current $I$, and at a height $h$ above a 1-D layered earth. At an observation point $z$ above the ground and 


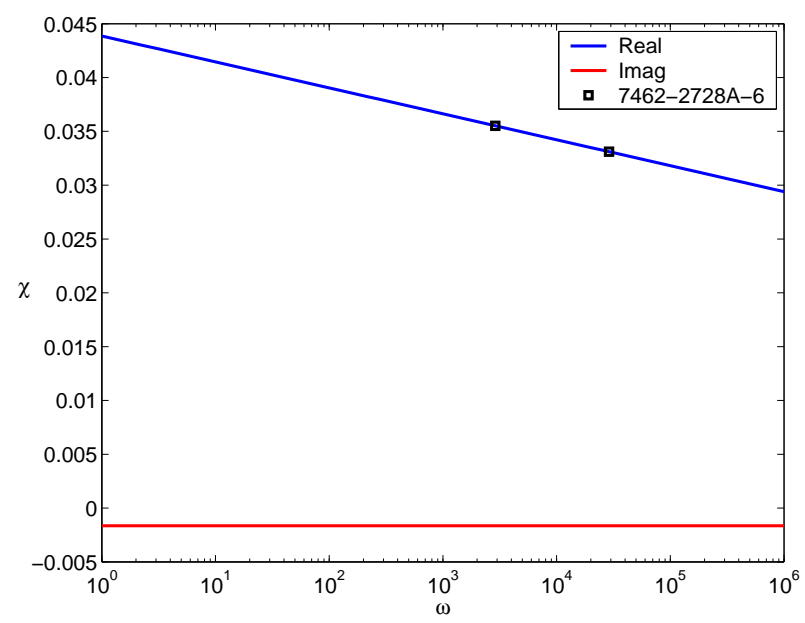

Figure 2: A magnetic susceptibility model constructed for soil sample 7463-2726A-6 from the Seagull Site at Kaho'olawe, Hawaii.

a radial distance $\rho$ from the axis of circular transmitter loop, the vertical component $H_{z}$ and the radial component $H_{\rho}$ of the $\mathbf{H}$-field are

$$
\begin{aligned}
& H_{\rho}(\omega)=\frac{I a}{2} \int_{0}^{\infty}\left[e^{-u_{o}(z+h)}-\frac{P_{21}}{P_{11}} e^{u_{o}(z-h)}\right] \lambda J_{1}(\lambda a) J_{1}(\lambda \rho) d \lambda \\
& H_{z}(\omega)=\frac{I a}{2} \int_{0}^{\infty}\left[e^{-u_{o}(z+h)}+\frac{P_{21}}{P_{11}} e^{u_{o}(z-h)}\right] \frac{\lambda^{2}}{u_{o}} J_{1}(\lambda a) J_{o}(\lambda \rho) d \lambda
\end{aligned}
$$

where $u_{o}=\sqrt{\lambda^{2}-k_{o}^{2}}, k_{o}$ is the wave number of the air, and $J_{o}$ and $J_{1}$ are the zeroth and first order Bessel functions, respectively. $P_{21}$ and $P_{11}$ are elements of the matrix $\mathbf{P}$ :

$$
\mathbf{P}=\mathbf{M}_{1} \prod_{j=2}^{m} \mathbf{M}_{j}
$$

where

$$
\begin{aligned}
& \mathbf{M}_{1}=\left[\begin{array}{cc}
\frac{1}{2}\left(1+\frac{\mu_{0} u_{1}}{\mu_{1} u_{0}}\right) & \frac{1}{2}\left(1-\frac{\mu_{0} u_{1}}{\mu_{1} u_{0}}\right) \\
\frac{1}{2}\left(1-\frac{\mu_{0} u_{1}}{\mu_{1} u_{0}}\right) & \frac{1}{2}\left(1+\frac{\mu_{0} u_{1}}{\mu_{1} u_{0}}\right)
\end{array}\right] \\
& \mathbf{M}_{j}=\left[\begin{array}{cc}
\frac{1}{2}\left(1+\frac{\mu_{j-1} u_{j}}{\mu_{j} u_{j-1}}\right) & \frac{1}{2}\left(1-\frac{\mu_{j-1} u_{j}}{\mu_{j} u_{j-1}}\right) \\
\frac{1}{2}\left(1-\frac{\mu_{j-1} u_{j}}{\mu_{j} u_{j-1}}\right) e^{-2 u_{j-1} t_{j-1}} & \frac{1}{2}\left(1+\frac{\mu_{j-1} u_{j}}{\mu_{j} u_{j-1}}\right) e^{-2 u_{j-1} t_{j-1}}
\end{array}\right]
\end{aligned}
$$

The thickness of the $j^{t h}$ layer is $t_{j}$, and $\mu_{j}$ is the magnetic permeability of the layer.

To determine the fields at the center of the transmitter loop, we can simply set $\rho=0$ and set $z=-h$. These substitutions give

$$
H_{\rho}(\omega)=0
$$




$$
H_{z}(\omega)=\frac{I a}{2} \int_{0}^{\infty}\left[1+\frac{P_{21}}{P_{11}} e^{-2 u_{o} h}\right] \frac{\lambda^{2}}{u_{o}} J_{1}(\lambda a) d \lambda
$$

Therefore, as the symmetry of the 1-D would also suggest, there is no horizontal component to the $\mathbf{H}$ field response at the center of the transmitter loop. This is an important point because it shows, for the situation where the fields are measured along the axis of the transmitter loop, that the effects of magnetic susceptibility will appear only in the vertical component. This feature will be exploited later when processing electromagnetic data.

The time domain solution is obtained by calculating Fourier transformations of the frequency response for a causal step turn-off [Newman et al., 1986]:

$$
\begin{aligned}
\frac{\partial h(t)}{\partial t} & =-\frac{2}{\pi} \int_{0}^{\infty} \operatorname{Re}[H(\omega)] \cos (\omega t) d \omega \\
\frac{\partial h(t)}{\partial t} & =\frac{2}{\pi} \int_{0}^{\infty} \operatorname{Im}[H(\omega)] \sin (\omega t) d \omega \\
h(t) & =-\frac{2}{\pi} \int_{0}^{\infty} \frac{\operatorname{Im}[H(\omega)]}{\omega} \cos (\omega t) d \omega
\end{aligned}
$$

These integrals are evaluated using the digital filters of Anderson [1975].

Let us consider a pair of two-layer models. The first, shown in Figure 3(a), consists of a $1 \mathrm{~m}$ thick top layer with a conductivity of $\sigma=0.01 \mathrm{~S} / \mathrm{m}$ and a frequency dependent complex susceptibility defined by Figure 2. The second two-layer model, shown in Figure 3(b), has a static real susceptibility of $\chi=0.05 \mathrm{SI}$ in the top layer. Both models have a basement conductivity of $\sigma=0.001 \mathrm{~S} / \mathrm{m}$ and a static susceptibility of $\chi=0.03$ SI. The calculated responses of this two-layer model will be compared to the response of a conductive and permeable sphere with the approximate material properties of steel $\left(\mu=150 \mu_{o}, \sigma=3.54 \times 10^{6} \mathrm{~S} / \mathrm{m}\right)$, a diameter of $25 \mathrm{~cm}$, and buried at a depth of $30 \mathrm{~cm}$. The sphere response will be approximated using the solution outlined by Ward [1959] for a sphere in a uniform field in a whole-space.

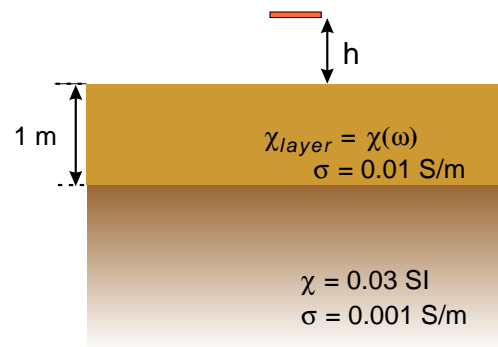

(a) Two layer model with frequency dependent magnetic susceptibility $\chi(\omega)$ in the top layer.

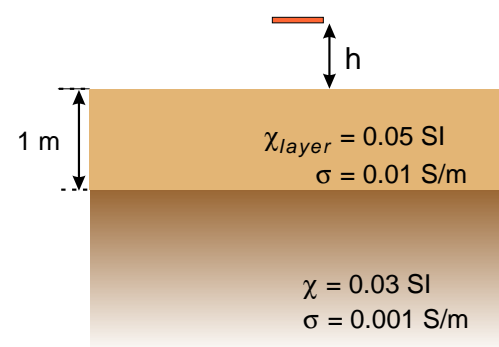

(b) Two layer model with static magnetic susceptibility.

Figure 3: Three example models.

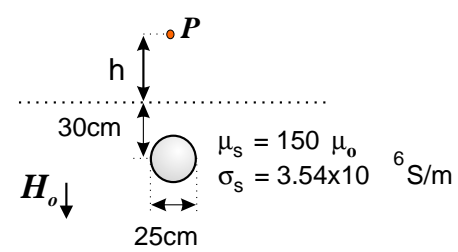

(c) A conductive and permeable metal sphere. Material parameters are chosen to match steel. 


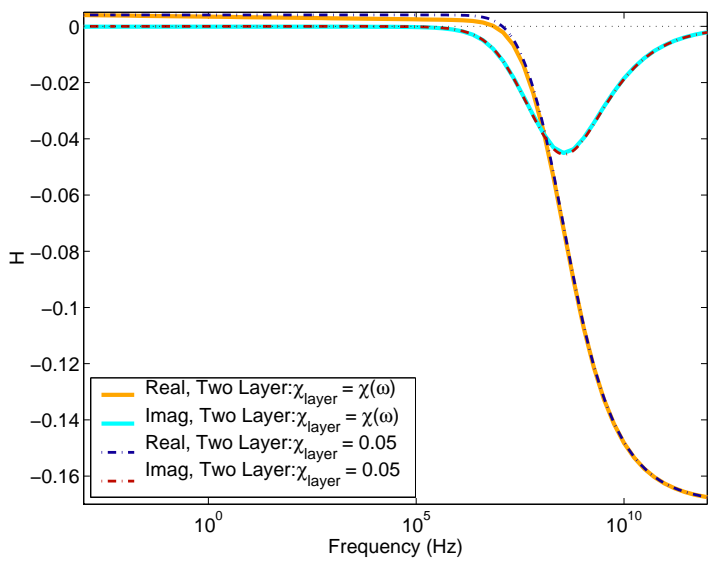

(a) The solid lines represent the response of the two layer model with frequency dependent susceptibility (Fig. 3(a)) and dashed lines represent the two layer

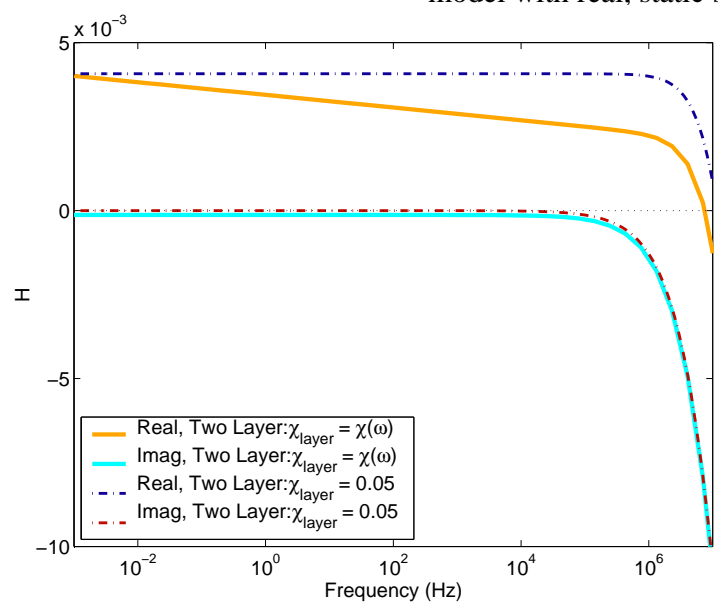

(b) Frequency domain response of the two layer model between $10^{-3}$ and $10^{7} \mathrm{~Hz}$.

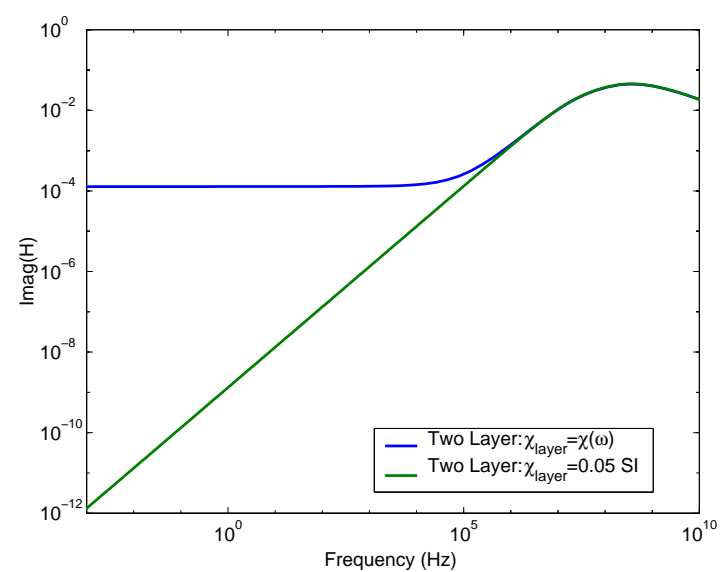

(c) The imaginary part of $\mathbf{H}$-field for the two layer model with frequency dependent susceptibility and with real static susceptibility.

Figure 4: Forward modelled frequency domain electromagnetic responses for the two layer model.

Figure 4(a) shows the modelled frequency response for the two-layer models. When plotting the responses at the chosen axis ranges, the results for the static, real susceptible model and the model with complex susceptibility look similar. Subtle, but important, differences become apparent when focusing the plot on the lower frequency range. Figure 4(b) shows that the real part of the $\mathbf{H}$-field has a line of negative slope. The effect of the frequency dependent layer on the quadrature component becomes evident when plotting the logarithm of the quadrature component (Figure 4(c)).

The effect of these differences on the time domain signature is shown in Figure 5(a). We see that the 


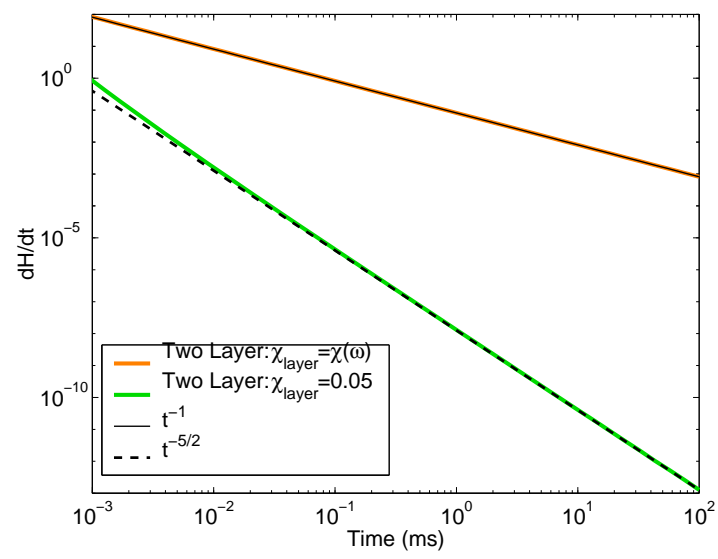

(a) Time domain responses of the two layer models. The model with frequency dependent susceptibility follows the $t^{-1}$ response while the model with only static, real susceptibility follows a $t^{-5 / 2}$ decay.

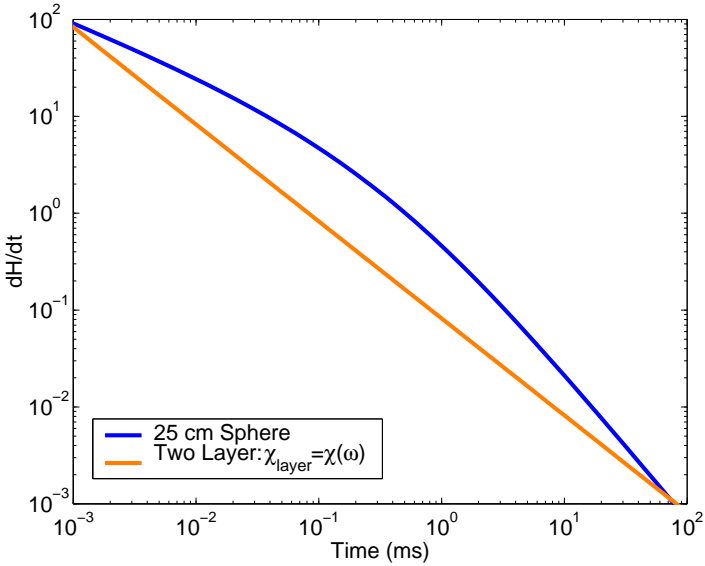

(b) Time domain responses of the the steel sphere and the two-layer model with frequency dependent susceptibility.

Figure 5: Forward modelled electromagnetic responses for the two layer model and the sphere model.

two layer model with complex, frequency dependent susceptibility produces a $t^{-1}$ response, while the two-layer model with static real susceptibility follows a $t^{5 / 2}$ decay. For the time range of the Geonics EM63 (approximately $0.1 \mathrm{~ms}$ to $25 \mathrm{~ms}$ ) it is clear that the response of the real, static susceptibility distribution produces a much weaker response than when the model contains the complex, frequency dependent susceptibility layer. Figure 4(d) shows the response of the sphere model is much larger than the TEM response of the model with only static, real susceptibility. However the TEM response for the model with complex susceptibility becomes comparable at early and late times.

The $t^{-1}$ response only occurs for a step function primary field. To take into account the finite length of the inducing field we can convolve the solution with the transmitter current [Asten, 1987]. The full waveform convolution is

$$
h_{R}(t)=\int_{0}^{\infty} h\left(t^{\prime}\right) h_{T}\left(t-t^{\prime}\right) d t^{\prime}
$$

where the $h_{R}(t)$ is the receiver waveform and $h_{T}(t)$ is the transmitter waveform. Figure 6(a) demonstrates how, as the pulse length increases, the $\partial H / \partial t$ response approaches the $t^{-1}$ behaviour of the step off response. From Figure 6(a), we see that to observe the $t^{-1}$ response at $10 \mathrm{~ms}$ after turn-off, the pulse length would need to be greater than $10 \mathrm{~ms}$.

\section{The Effect of Susceptibility on Dipole Discrimination Algorithms}

In this section we consider the effect of magnetic soils on the ability to recover the representative dipole parameters of a buried target. We consider data from a 3-component time domain sensors. 


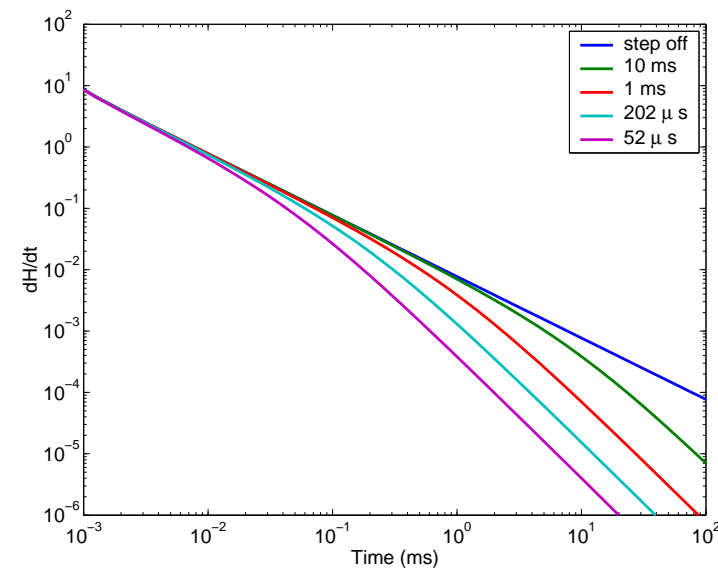

(a) Two layer model

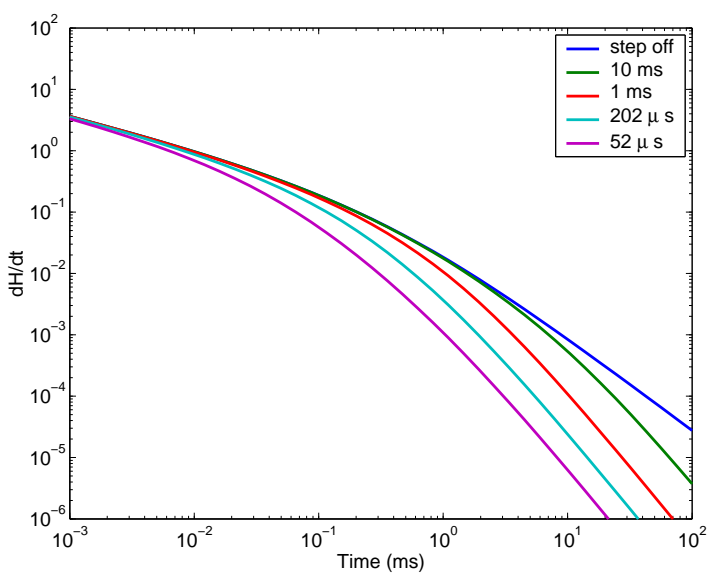

(b) Sphere model

Figure 6: Comparison of the the $\partial H / \partial t$ responses for different transmitter loop heights and pulse lengths.

\section{Generation of Synthetic TEM Data}

Although 3-component sensors have been developed by Geonics Inc. (EM61-3D) and Zonge Engineering (NanoTEM), testing of either sensor has been limited. Field data, in particular data acquired over magnetic soils, are not readily available. Therefore, to investigate the effects of the magnetic soils we must generate synthetic data sets. We generate synthetic field data sets by assuming that the secondary field is a linear sum of the response of the buried metallic target, the response of the magnetic soil and Gaussian noise:

$$
d_{x}=F_{x}^{d}[\mathbf{m}]+\epsilon, \quad d_{y}=F_{y}^{d}[\mathbf{m}]+\epsilon, \quad d_{z}=F_{z}^{d}[\mathbf{m}]+F^{s o i l}+\epsilon
$$

where $d_{x}, d_{y}$, and $d_{z}$ are the three components of secondary field recorded by the sensor, $F_{x}, F_{y}$, and $F_{z}$ are components of the buried metal target response in the absence of the magnetic soil, $F^{s o i l}$ is the response of the magnetic soils and $\epsilon$ is the Gaussian noise. We have used the conclusion, arrived at earlier, that the magnetic soils affect only the verified component of the receiver if the receiver is on the axis of the transmitter.

The buried target response $\mathbf{F}^{d}[\mathbf{m}]$ is calculated by using the decaying two-dipole approximation outline in Pasion and Oldenburg [2001a]. The response of a compact buried target can be approximated by a 13 element model vector:

$$
\mathbf{m}=\left[X, Y, d, \theta, \phi, k_{1}, \alpha_{1}, \beta_{1}, \gamma_{1}, k_{2}, \alpha_{2}, \beta_{2}, \gamma_{2}\right]
$$

where $(X, Y)$ is the target location, $d$ is the depth below the surface, $\theta$ and $\phi$ define the target orientation, $k_{1}, \alpha_{1}, \beta_{1}, \gamma_{1}$ define the decay characteristic of a dipole oriented parallel to the axis of symmetry of the target, and $k_{2}, \alpha_{2}, \beta_{2}, \gamma_{2}$ define the decay characteristic of a dipole oriented perpendicular to the axis of symmetry of the target. The validity of this model has verified, as well as appropriate parameters for different UXO and scrap targets, are reported in Pasion and Oldenburg [2001b]. For the examples in this report, we will forward model the response for the stokes mortar of Figure 7(a). The stokes mortar will 


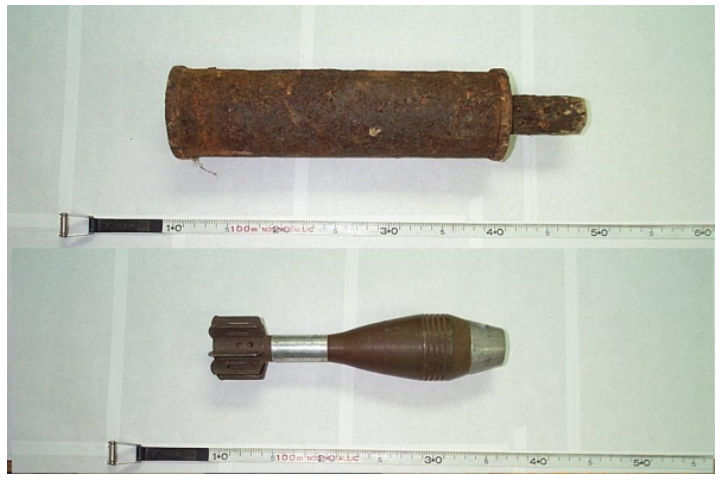

(a) Photos of a Stokes mortar (top) and a $60 \mathrm{~mm}$ mortar.

\begin{tabular}{|l||c|c|}
\hline $\begin{array}{l}\text { Decay } \\
\text { Parameter }\end{array}$ & $\begin{array}{l}\text { Stokes } \\
\text { Mortar }\end{array}$ & $\begin{array}{c}60 \mathrm{~mm} \\
\text { Mortar }\end{array}$ \\
\hline \hline$k_{1}$ & 43.9 & 3.38 \\
\hline$\alpha_{1}$ & 0.02 & 0.019 \\
\hline$\beta_{1}$ & 0.73 & 0.9 \\
\hline$\gamma_{1}$ & 9.1 & 2.55 \\
\hline \hline$k_{2}$ & 4.9 & 0.79 \\
\hline$\alpha_{2}$ & 0.001 & 0.02 \\
\hline$\beta_{2}$ & 1.09 & 1.19 \\
\hline$\gamma_{2}$ & 10.8 & 3.59 \\
\hline
\end{tabular}

(b) Characteristic decay constants for the Stokes and $60 \mathrm{~mm}$ mortar.

Figure 7: Photos and Decay Constants for a Stokes mortar and a $60 \mathrm{~mm}$ mortar.

be placed at $(X, Y)=(2 \mathrm{~m}, 2 \mathrm{~m})$ and at a depth of $40 \mathrm{~cm}$. The target will be oriented such that $(\theta, \phi)=(30$ degrees, 70 degrees).

We also showed that an appropriate representation for the magnetic soil response is

$$
F^{\text {soil }}=A t^{-1}
$$

The magnitude of the soil signal is varied throughout the survey area by adding a random component to $A$ at each station location, that is

$$
F^{\text {soil }}=\left(\bar{A}+\epsilon_{A}\right) t^{-1}
$$

where $\bar{A}$ controls the overall level of the soil signal and $\epsilon_{A}$ is Gaussian distributed random noise that has a standard deviation equal to $25 \%$ of $\bar{A}$. Two different soil signal distributions are considered in this report. For the first distribution $\bar{A}$ is chosen such that at the first time channel of the EM63 response (180 $\mu s$ ) the mean soil signal over the survey area is $50 \mathrm{mV}$. For the second distribution the mean soil signal is set to $100 \mathrm{mV}$. Figures 8 (a) and (b) plots the distribution of decay curve for the two different choices of $\bar{A}$ (labelled $\bar{A}=A^{50 m V}$ and $\bar{A}=A^{100 m V}$, respectively). Figure 8(c) compares the mean soil signal $A^{50 m V} t^{-1}$ and $A^{100 m V} t^{-1}$ with the response of the Stokes mortar measured directly over the target (i.e. at $(X, Y)=(2 \mathrm{~m}, 2 \mathrm{~m}))$. Figure $8(\mathrm{c})$ shows that the response of the Stokes mortar has a portion of its curve that is simlar to the $t^{-1}$ response of the magnetic soil. This similarity makes the subtraction of the magnetic soil response from survey data more difficult.

Finally some noise $\epsilon$ is added to the sum of the basalt response and the dipole response. The amount of noise has a standard deviation of $5 \%$ of the data plus $0.5 \mathrm{mV}$.

\section{Pre-processing of Data Using Horizontal Components of Field}




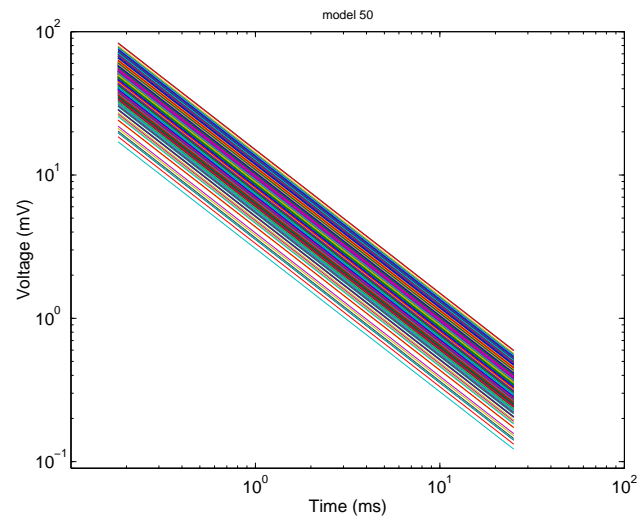

(a) Distribution of basalt models $F^{\text {soil }}=$ $\left(A^{50 m V}+\epsilon_{A}\right) t^{-1}$.

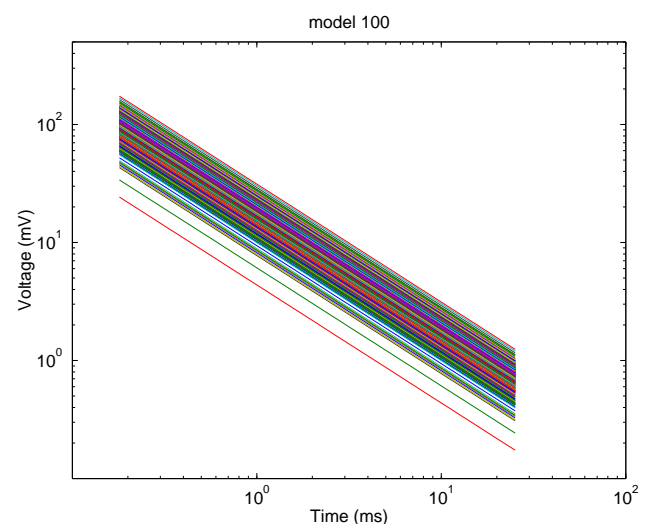

(b) Distribution of basalt models $F^{\text {soil }}=$ $\left(A^{100 m V}+\epsilon_{A}\right) t^{-1}$.

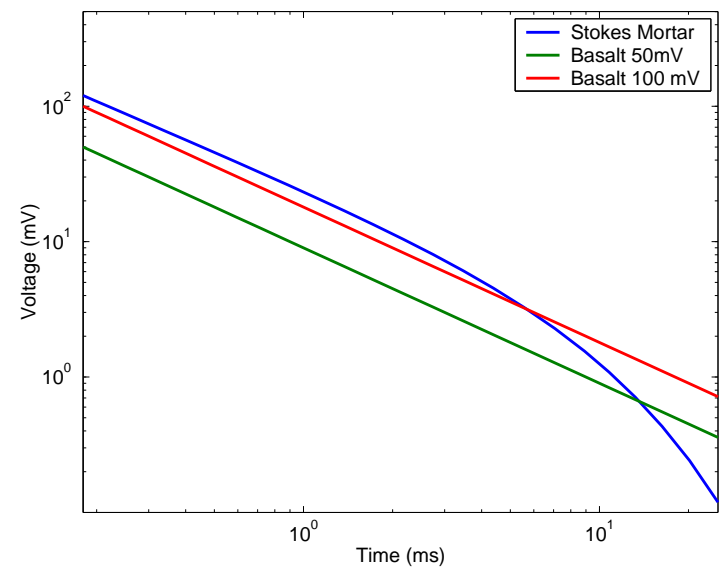

(c) Comparison of the decay response directly above a Stokes mortar buried $40 \mathrm{~cm}$ deep, $F^{\text {soil }}=$ $A^{50 m V} t^{-1}$ and $F^{s o i l}=A^{100 m V} t^{-1}$.

Figure 8: Different Basalt models.

As our modelling suggests, the presence of magnetic soils will produce a $t^{-1}$ signal in the vertical component of the measured secondary field. The difficulty in removing this signal lies in identifying if the measured response is only from the soil or if the response is partly due to the presence of a metallic target. The absence of the soil signal in the horizontal components suggest that they can be used as part of a pre-processing step to help determine where and how we should attempt to remove the soil signal in the vertical component. One possible (and simple) way of doing this would be to

1. Calculate the horizontal component of the data at the first time channel: $d^{h}\left(t_{1}\right)=\sqrt{d_{x}^{2}\left(t_{1}\right)+d_{y}^{2}\left(t_{1}\right)}$.

2. At each sounding, if $d^{h}$ is less than some threshold value (e.g. $d^{h}<2 \mathrm{mV}$ ) then identify this station as not likely containing signal from a target. We can proceed to fit $A t^{-1}$ to the data at this station 


\begin{tabular}{|l||c|c|c|c|c|c|}
\hline & $\begin{array}{c}\text { Real } \\
\text { Parameters }\end{array}$ & No Basalt & $\begin{array}{c}\text { 50mV Basalt } \\
\text { Model }\end{array}$ & $\begin{array}{c}\text { 50mV Basalt } \\
\text { Model,Basalt } \\
\text { Response } \\
\text { subtracted }\end{array}$ & $\begin{array}{c}\text { 100mV Basalt } \\
\text { Model }\end{array}$ & $\begin{array}{c}\text { Model, Basalt } \\
\text { Response } \\
\text { subtracted }\end{array}$ \\
\hline \hline$X$ & 2 & 2 & 2 & 1.98 & 1.78 & 1.7 \\
\hline$Y$ & 2 & 2 & 2.02 & 1.99 & 1.84 & 1.77 \\
\hline depth & 0.4 & 0.4 & 0.37 & 0.39 & 0.35 & 0.35 \\
\hline$\phi$ & 30 & 30.1 & 31.9 & 29.9 & 26.2 & 51.8 \\
\hline$\theta$ & 70 & 69.9 & 72.5 & 65.9 & 164.6 & 180 \\
\hline \hline$k_{1}$ & 43.9 & 45.113 & 47.455 & 39.866 & 11.243 & 11.239 \\
\hline$\alpha_{1}$ & 0.02 & 0.001 & 0.019 & 0.019 & 0.001 & 0.018 \\
\hline$\beta_{1}$ & 0.73 & 0.664 & 0.667 & 0.638 & 0.744 & 0.76 \\
\hline$\gamma_{1}$ & 9.1 & 6.442 & 5.998 & 7.379 & 12.924 & 10.869 \\
\hline \hline$k_{2}$ & 4.9 & 5.309 & 6.201 & 4.358 & 24.574 & 5.991 \\
\hline$\alpha_{2}$ & 0.001 & 0.001 & 0.019 & 0.019 & 0.001 & 0.018 \\
\hline$\beta_{2}$ & 1.09 & 1.038 & 1.03 & 1.073 & 0.657 & 0.904 \\
\hline$\gamma_{2}$ & 10.8 & 5.566 & 6.549 & 5.361 & 25.101 & 19.769 \\
\hline \hline
\end{tabular}

Table 2: Inversion Results for a Stokes Mortar buried in a background of magnetic soils.

and subtract from the data.

3. At soundings where $d^{h}$ is greater than the threshold, we can then subtract $A^{\dagger} t^{-1}$ where $A^{\dagger}$ is determined from the fitting of stations in the previous step. For example, $A^{\dagger}$ could be the mean value of all the previously obtained $A$ values, or we could interpolate values for $A^{\dagger}$ from the $A$ values.

In the examples of the final section, $A^{\dagger}$ is calculated by taking the mean value of the $A$ values obtained from data where $d^{h}<2 \mathrm{mV}$.

\section{Inversion Results}

The synthetic data were inverted for the 13 parameters listed in equation (17). These parameters were obtained by minimizing a least squares objective function in two steps. The first step was to minimize the objective function using a global optimization algorithm, and the second step was to take the result from the global algorithm and use it as a starting point for a local algorithm. We used the neighbourhood algorithm [Sambridge, 1999] for the global search, and a Projected BFGS algorithm [Kelley, 1999] for the local search.

The synthetic data was generated for a Stokes Mortar buried at a depth of $40 \mathrm{~cm}$ and located at $(X, Y)=(2 \mathrm{~m}, 2 \mathrm{~m})$. Three different magnetic soil models were used: $\bar{A}=0, A^{50 m V}$, and $A^{100 m V}$. The results of inverting the data sets are found in table 2. Table 2 show that the inversion was reasonably successful in recovering the location, orientation, and dipole parameters in all cases except for when the basalt signal was $F^{s o i l}=\left(A^{100 m V}+\epsilon_{A}\right) t^{-1}$. Figure 9 examines the data fit when the magnetic 
soil response is $F^{\text {soil }}=\left(A^{50 m V}+\epsilon_{A}\right) t^{-1}$. Even though the basalt was not removed for this inversion, the algorithm was very successful in recovering the model parameters due to presence of the horizontal components.

\section{Conclusions}

Electromagnetic sensor data acquired in the presence of magnetic soils has a characteristic $\partial H / \partial t$ decay of $t^{-1}$. This $t^{-1}$ decay can be can be derived by assuming the soil consists of magnetic particles with a uniform distribution of decay times. This assumption leads to a representation of the magnetic susceptibility as a frequency dependent, complex quantity. The 1D forward modelling of this susceptibility model reveal that the horizontal components of data measured along the axis of a transmitter loop is not sensitive to magnetic soils provided that the subsurface properties can be adequately represented by a 1D layered model. As a consequence, when inverting the three component sensor data collected over a target buried magnetic soil, the information provided by the horizontal components of data may: (1) improve detection, (2) be exploited in developing processing routines to aid in the removal of the magnetic soil response in the vertical component of data, and (3) help constrain the inversion to more reliably recover dipole model parameters.

\section{Acknowledgments}

We would like to thank Dwain Butler from the Engineer Research and Development Center for the magnetic susceptibility measurements of Kaho'olawe, Hawaii soils. 


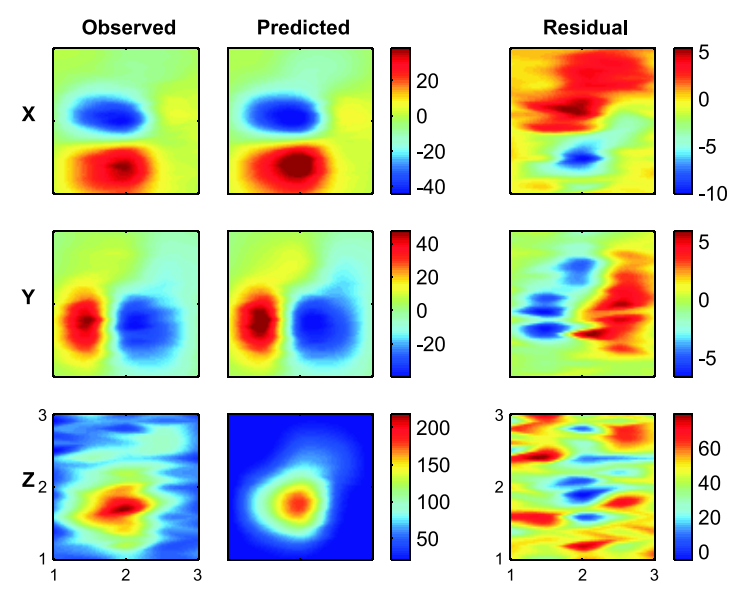

(a) Plan view of observed and predicted data and residual for the first time channel $(t=180 \mu \mathrm{s})$.
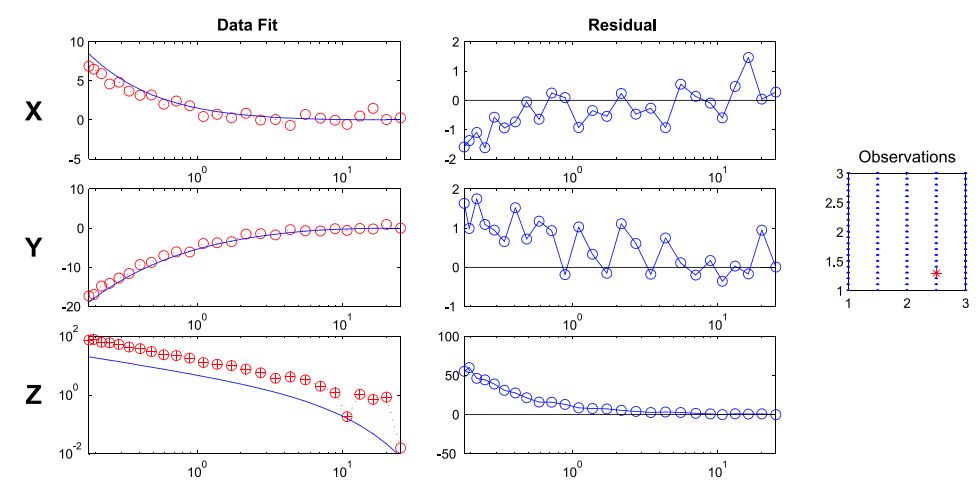

(b) Decay curve comparison at $(X, Y)=(2.5,1.3) \mathrm{m}$.
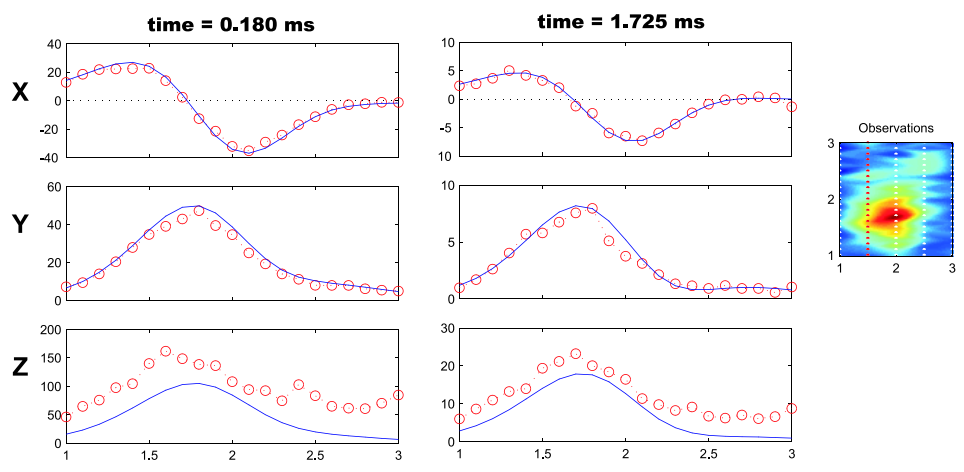

(c) Profile of observed and predicted data and residual for the line $X=$ $1.5 \mathrm{~m}$.

Figure 9: Inversion results for a data set with a basalt background of $F^{\text {soil }}=A^{50 m V} t^{-1}$. The bias in the $Z$ component indicates the inability to fit the basalt background due to the presence of the horizontal components of the data. 


\section{References}

[Asten, 1987] Asten, M. W., 1987, Full transmitter waveform transient electromagnetic modeling and inversion for soundings over coal measures: Geophysics, 47, 1315-1324.

[Buselli, 1982] Buselli, G., 1982, The effect of near surface superparamagnetic material on electromagnetic measurements: Geophysics, 47, 1315-1324.

[Carmichael, 1989] Carmichael, R.S., 1989, CRC Practical Handbook of Physical Properties of Rocks and Materials: CRC Press, Boca Raton, Fla.

[Cespedes, 2001] Cespedes, E.R., 2001, Demonstration of Advanced Unexploded Ordnance Detection and Discrimination Technologies at Kaho'olawe, Hawaii: Proceedings of Partners in Environmental Technology Techinical Symposium and Workshop, Washington, D.C.

[Chikazumi, 1997] Chikazumi, S., 1997, Physics of Ferromagnetism, 2nd ed.: Oxford University Press.

[Colani and Aitken, 1996] Colani, C. and Aitken, M.J., 1966, Utilization of Magnetic Viscosity Effects in Soils for Archaeological Prospection: Nature, 212, 1446-1447.

[Dabas et al., 1992] Dabas, M., Jolivet, J., and Tabbagh, Al, 1992, Magnetic Susceptibility and Viscosity of Soils in a Weak Time Varying Field: Geophysical Journal International, 108, 101-109.

[Farquharson et al., 2001] Farquharson, C.G., D.W. Oldenburg and Partha S. Routh. Simultaneous onedimensional inversion of loop-loop electromagnetic data for both magnetic susceptibility and electrical conductivity, submitted to Geophysics.

[Kelley, 1999] Kelley, S., 1999, Iterative Methods fo Optimization. Society for Industrial and Applied Mathematics, Philadelphia, PA.

[Olhoeft and Strangway, 1974] Olhoeft, G.R. and Strangway, D.W., 1974, Magnetic Relaxation and the Electromagnetic Response Parameter: Geophysics, 39, no.3, 302-311.

[Mullins and Tite, 1974] Mullins, C.E. and Tite, M.S., 1974, Magnetic susceptibility and frequency dependence of susceptibility in single-domain assemblies of magnetite and maghemite: J. Geophys. Res, 78, 804-809.

[Newman et al., 1986] Newman, G.A., Hohmann, G.W., and Anderson, W.L., 1986, Transient electromagnetic response of a three-dimensional body in a layered earth: Geophysics, 51, no.8, 16081627.

[Parsons-UXB, 1998] Parsons Engineering and UXB International Inc., 1998, Cleanup Plan: UXO Clearance Project, Kaho'olawe Island Reserve, Hawaii. Prepared for Naval Facillities Engineering Command Pacific Division.

[Pasion and Oldenburg, 2001a] Pasion, L.R. and Oldenburg, D.W., 2001, A Discrimination Algorithm for UXO Using Time Domain Electromagnetics: Journal of Engineering and Environmental Geophysics, 6, no.2, 91-102. 
[Pasion and Oldenburg, 2001b] Pasion, L.R. and Oldenburg, D.W., 2001, Locating and Characterizing Unexploded Ordnance Using Time Domain Electromagnetic Induction, Technical Report ERDC/GSL TR-01-10, U.S. Army Research and Development Center, Vicksburg, MS.

[Lee, 1983] Lee, T., 1983, The Effect of a Superparamgnetic Layer on the Transient Electromagnetic Response of a Ground: Geophysical Prospecting, 32, 480-496.

[Sambridge, 1999] Sambridge, M., 1999, Geophysical Inversion with a Neighbourhood Algorithm -I. Searching a parameter space: Geophys. J. Int., 138,479-494.

[Stearns, 1940] Stearns, H. T. 1940. Geology of Ground Water Resources on the Islands of Lanai and Kaho'olawe, Hawaii, Bulletin 6. Hawaii Division of Hydrography, Honolulu, Hawaii.

[Scollar et al., 1990] Scollar, I., Tabbagh, A., Hesse, A., and Herzog, I., 1990. Archaeological Prospecting and Remote Sensing. Cambridge University Press, Cambridge.

[Ward, 1959] Ward, S.H., 1959, Unique Determination of Conductivity, Susceptibility, Size, and Depth in Multifrequency Electromagnetic Exploration: Geophysics, 24, no.3, 531-546.

[Ward and Hohmann, 1991] Ward, S.H.., and Hohmann, G.W., 1991, Electromagnetic Theory for Geophysical Applications, in Nabighian, M.N., Ed., Electromagnetic Methods In Applied Geophysics: Society of Exploration Geophysicists, Volume 1, Theory, 131-311.

[Ware, 2001] Ware, G.H., 2001, EM-63 Decay Curve Analysis for Ordnance Discrimination: Proceedings of Partners in Environmental Technology Techinical Symposium and Workshop, Washington, D.C. 